\title{
A single-layer micromachined tunable capacitor with an electrically floating plate
}

\author{
Fahimullah Khan, Yong Zhu, Junwei Lu, Jitendra Pal, Dzung Viet Dao \\ School of Engineering, Griffith University, 4222, Queensland, Australia \\ Queensland Micro- and Nanotechnology Centre, Griffith University, QLD, 4111, Australia \\ Email: y.zhu@griffith.edu.au
}

\begin{abstract}
:
This paper reports a novel micromachined tunable capacitor with an electrically floating movable plate. The device has been fabricated from a single metal layer based on a low-cost standard process. Tunable capacitors with electrically floating and non-floating plates have been characterized and compared with each other by using the same fabricated device with different configurations. The floating tunable capacitor exhibits a higher quality factor (Q-factor) compared to non-floating capacitor by eliminating the spring's resistance loss in radio frequency (RF) signal path. The device is actuated by an electro-thermal actuator to achieve high capacitance tuning range without the pull-in effect issue existed in parallel-plate electrostatic actuators. Experimental results show that the tunable capacitor has a wide capacitance tuning range of $631 \%$ with a low actuation voltage of $0.72 \mathrm{~V}$. The floating tunable capacitor has a Q-factor at $1 \mathrm{GHz}$ of 24.4 , which is 5.5 times higher than that of non-floating traditional tunable capacitor fabricated on the same chip.
\end{abstract}

\section{Introduction}

Radio frequency MEMS (RF MEMS) provides attractive capabilities in switching and tuning systems compared to conventional varactor diodes in applications such as tunable filters, oscillators and phase shifters. The most important parameters for those devices are Q-factor and tuning range [1-3]. Conventional MOS capacitors and varactor diodes have been used for tuning the channel bandwidth. However, these devices have limited frequency tuning range and high phase noise due to their non-linearity errors. For example, the phase noise of an oscillator is directly proportional to $1 / \mathrm{Q}^{2}$, where $\mathrm{Q}$ is the quality factor of the oscillator [4]. Therefore, high Q-factor is desired to reduce phase noise. Compared with semiconductor varactors, MEMS tunable capacitors can provide higher quality factor, wider tuning range, higher linearity and lower loss in a small foot print $[5,6]$.

In traditional MEMS tunable capacitors [2, 6, 7], to achieve high tuning range, the suspension beams have to be narrow, long and soft for low voltage or current actuation requirements. However, soft beams contribute significant resistance loss for RF signal [8]. Therefore, there is a trade-off between the actuation voltage/current and Q-factor if the RF signal is passing through the suspension beams. To improve the quality factor, a new type of tunable capacitor with electrically floating plate has been introduced [9, 10]. The Q-factor has been improved by eliminating the RF signal in suspension beams. However, the design has low tuning ratio (41\%) due to pull-in effect in parallel plate electrostatic actuator [10, 11]. In order to reduce the fabrication complexity, a floating tunable capacitor with a single structure layer and in-plane movement was reported by our group [9], where the Q-factor was derived based on theoretical calculation.

In this paper, a single-layer micromachined tunable capacitor with an electrically floating plate is introduced and fabricated in a low-cost Metal-MUMPs ${ }^{\mathrm{TM}}$ process. For a fair comparison purpose, floating and non-floating capacitors are characterized and compared using the same fabricated device with different configurations. With no resistance loss in the suspension beams, a higher Q-factor of 24.4 at $1 \mathrm{GHz}$ has 
been achieved in floating capacitor. Unlike parallel plate electrostatic actuator, which has the pull-in effect, electro-thermal actuator can provide large displacement and strong force at low driving voltage [12, 13], therefore a high capacitance tuning range of $631 \%$ has been obtained.

\section{Device design}

To eliminate spring's resistance loss in radio frequency (RF) signal path, electrically floating plate tunable capacitor concept is adopted in this paper. Figure 1 illustrates the conceptual schematic view of the tunable capacitor with an electrically floating plate. The gap between the fixed plates $\mathrm{A} / \mathrm{B}$ and movable plate $\mathrm{C}$ can be adjusted by moving the floating plate $C$ through the Chevron-shaped thermal actuator. The RF signal flows from plate A through floating plate $\mathrm{C}$ (not grounded) to plate B as shown in figure 1. As RF signal does not go through the suspension beam, resistance loss in traditional tunable capacitors can be eliminated.

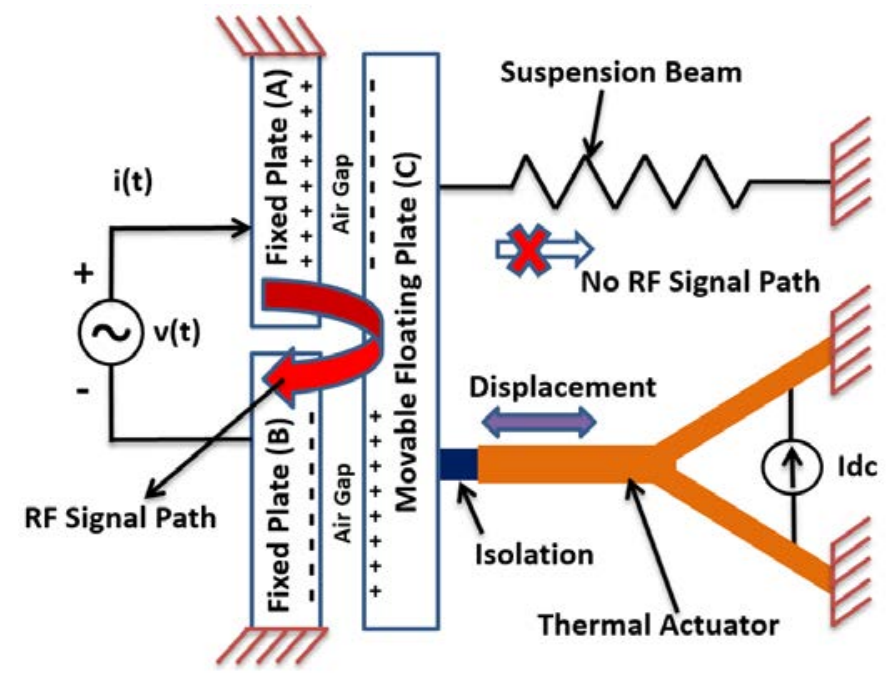

Figure 1. Conceptual schematic view of the tunable capacitor with Chevron-shaped electro-thermal actuator and electrically floating plate.

Due to the pull-in effect in parallel plate actuator, limited capacitance tuning range of $50 \%$ was obtained [14]. Lateral comb drive can achieve a controllable displacement actuation without pull-in effect. However, it requires high voltage for large capacitance tuning range[9, 15]. Electro-thermal actuator can produce large forces and rectilinear displacement caused by Joule's heating effect with low voltage [13, 16]. Therefore, electro-thermal actuation can be selected for changing the gap between capacitor plates for the applications where low-voltage actuation is required. As illustrated in figure 2, the Chevron-shaped actuator beams have an initial angle of $1.05^{\circ}$ and are made of nickel material.

The proposed tunable capacitor consists of transverse comb structures, including fixed and movable comb fingers. Movable comb fingers are mechanically supported by folded suspension beams and electro-thermal actuators as shown in figure 2 . A $700 \mathrm{~nm}$ silicon nitride $\left(\mathrm{Si}_{3} \mathrm{~N}_{4}\right)$ layer mechanically connects and electrically isolates the movable comb fingers and electro-thermal actuator. As dc and ac signal paths are isolated, there is no need for bias tees or electronic circuitry to separate the two signals. The detailed structure parameters and values of the tunable capacitor are listed in table 1. 


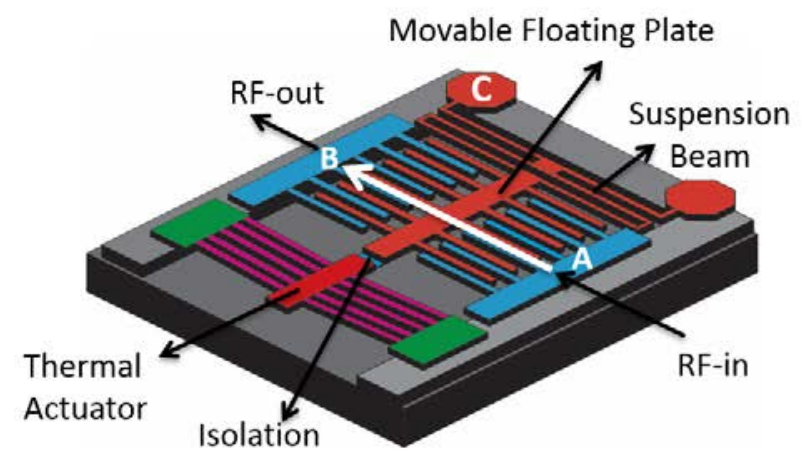

(a)

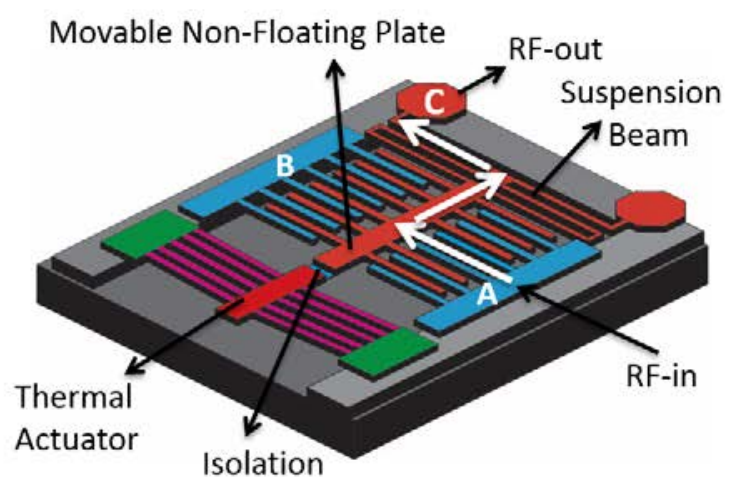

(b)

Figure 2. Schematic views for the comparison of tunable capacitors with different configurations using the same fabricated device: (a) in floating plate tunable capacitor, RF signal travels from point A through movable floating plate to point B with point $\mathrm{C}$ left electrically floating; (b) in conventional non-floating plate tunable capacitor, RF signal travels from point $A$ to point $C$ through narrow and long suspension beam, causing high resistance loss.

Table 1. Design parameters and values for tunable capacitor

\begin{tabular}{cc}
\hline \hline Parameter & Value \\
\hline Tunable capacitor initial gap $(\mu \mathrm{m})$ & 6 \\
Length of capacitor comb finger $(\mu \mathrm{m})$ & 480 \\
Width of capacitor comb finger $(\mu \mathrm{m})$ & 10 \\
Number of capacitor comb finger each side & 21 \\
Suspension beam width $(\mu \mathrm{m})$ & 8 \\
Suspension beam length each side $(\mu \mathrm{m})$ & $3 \times 450$ \\
Number of Chevron-shaped actuator beams & 5 \\
Initial angle of the actuator beams $\left({ }^{\circ}\right)$ & 1.05 \\
Length of actuator beams $(\mu \mathrm{m})$ & 1000 \\
Device thickness $(\mu \mathrm{m})$ & 20.5 \\
Air trench thickness $(\mu \mathrm{m})$ & 25 \\
\hline
\end{tabular}

The tuning range of capacitor is defined as the ratio of maximum capacitance change to the minimum capacitance, as given below:

$$
\text { Turning Range }=\frac{C_{\max }-C_{\min }}{C_{\min }} \times 100 \%
$$

where $C_{\max }$ is the maximum capacitance and $C_{\min }$ is the minimum capacitance. It is noted from the above equation that in order to increase the tuning range the change in capacitance should be as large as possible, which can be done by changing the gap between capacitor fingers as much as possible.

The second important parameter is the Q-factor, which is defined as the ratio of energy stored in the device to the energy dissipated in a full cycle. The capacitance $C$ and quality factor $Q$ of a capacitor can be obtained from the following equations:

$$
C=\frac{1}{2 \pi f|\operatorname{imag}\{Z\}|}
$$




$$
Q=\frac{1}{2 \pi f C(\operatorname{real}\{Z\})}=\frac{1}{2 \pi f C R_{s}}
$$

where $Z$ is the impedance of capacitor and $R_{s}$ is the series resistance of capacitor. The impedance of capacitor can be obtained from Scattering parameters (S-parameters) [17]:

$$
Z=Z_{o} \frac{2\left(1-S_{21}\right)}{S_{21}}
$$

where $S_{21}$ is the forward voltage gain of tunable capacitor and $Z_{0}$ is the characteristic impedance. Equation (3) implicates that the Q-factor of capacitor is inversely proportional to the series resistance of the capacitor. Therefore, the series resistance has to be reduced to increase the Q-factor. To minimize the series resistance in the RF signal path, the RF signal flows from point A to point B through floating plate, without passing through the narrow and long suspension beams, as shown by an arrow in figure 2(a). For a fair comparison purpose, figure 2(b) mimics the conventional tunable capacitor configuration by using the same device with different connections. In this configuration, RF signal flows from point A through the narrow and long suspension beams to point $\mathrm{C}$, as indicated by the arrows in figure 2(b). It is noted that by using this electrically floating plate technique, the series resistance is greatly reduced, which is validated by the experimental results given in the section of experimental results and discussions.

\section{Fabrication process}

The design was fabricated in a Metal-MUMPs ${ }^{\mathrm{TM}}$ standard process, which is a commercially available lowcost MEMS process [18]. The actuator and capacitor are made by nickel material, which has good electrical, mechanical and thermal properties as listed in table 2. Nickel has low electrical resistivity, thus lower resistance loss associated with capacitor combs can be achieved compared to the silicon-based capacitor in [9]. Also, nickel actuators are more energy efficient that silicon actuators because the coefficient of thermal expansion (CTE) of nickel is 5.7 times larger than that of silicon [19].

Table 2. Electrical, mechanical and thermal properties of nickel

\begin{tabular}{cc}
\hline \hline Parameter & Value \\
\hline Electrical resistivity $(\mathrm{ohm} \cdot \mathrm{cm})$ & $8 \times 10^{-6}$ \\
Young Modulus $(\mathrm{GPa})$ & 210 \\
Poisson ratio & 0.31 \\
Coefficient of thermal expansion $(\mathrm{ppm} / \mathrm{K})$ & 13 \\
Thermal conductivity $(\mathrm{W} / \mathrm{mK})$ & 91 \\
Plastic deformation temperature $\left({ }^{\circ} \mathrm{C}\right)$ & $\sim 350$ \\
\hline
\end{tabular}

Figure 3 illustrates the cross-sectional view of the tunable capacitor structure. To reduce the eddy current loss in the substrate at high frequencies, the device is made on a high electrical resistivity $(>4,000 \mathrm{ohm} \cdot \mathrm{cm})$ $\mathrm{N}$-type (100) silicon substrate with a $25 \mu \mathrm{m}$ air trench. The tunable capacitor and thermal actuator are made of $20 \mu \mathrm{m}$ nickel layer by electroplating process. The mechanical connection and electrical isolation between tunable capacitor and electro-thermal actuator are achieved by a $700 \mathrm{~nm}$ silicon nitride $\left(\mathrm{Si}_{3} \mathrm{~N}_{4}\right)$ layer. As the isolation layer is much thinner than the nickel structure layer, the silicon nitride layer may bend during actuation. Therefore, a U-shaped isolation structure is adopted to increase the strength of the isolator, as illustrated in the inset of figure 4. The SEM images of the final fabricated device are shown in figure 4. 


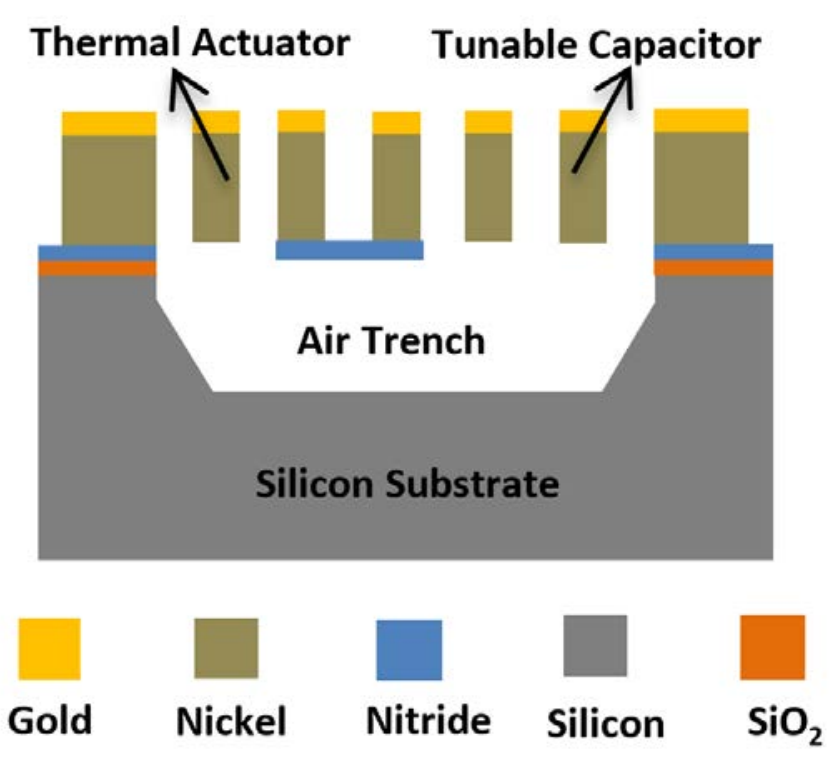

Figure 3. Cross-sectional view of the tunable capacitor structure, including high resistivity silicon substrate, $25 \mu \mathrm{m}$ air trench, $700 \mathrm{~nm}$ silicon nitride isolator, $20 \mu \mathrm{m}$ nickel device layer and $500 \mathrm{~nm}$ gold contact layer.

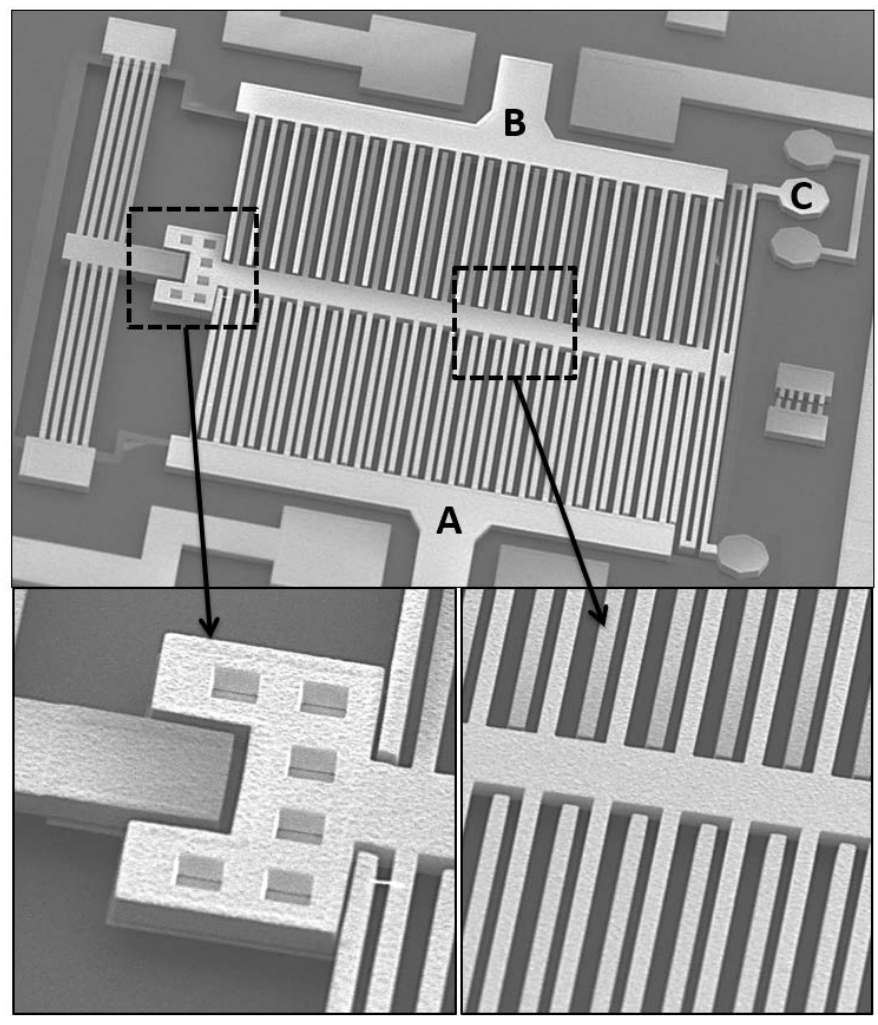

Figure 4. SEM images of the tunable capacitor. Ground-signal-ground (GSG) pads are adopted for accurate radio frequency measurement. A U-shaped isolator structure can increase the mechanical connection strength between tunable capacitor and thermal actuator. 


\section{Experimental results and discussions}

The experimental setup to test the characteristics of tunable capacitor consists of Cascade Micro-tech probe station, Rohde \& Schwarz ZVL vector network analyzer, high frequency (HF) ground-signal-ground (GSG) probes, $40 \mathrm{GHz}$ high frequency connecting cables, dc cables and dc power supply. The photographs of apparatus setup and probe connections are shown in figure 5.

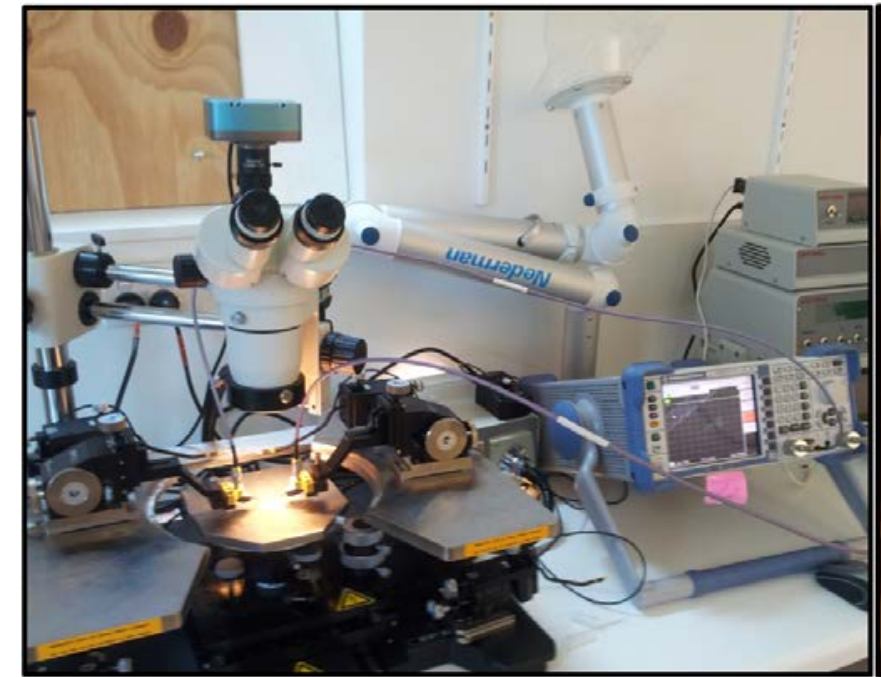

(a)

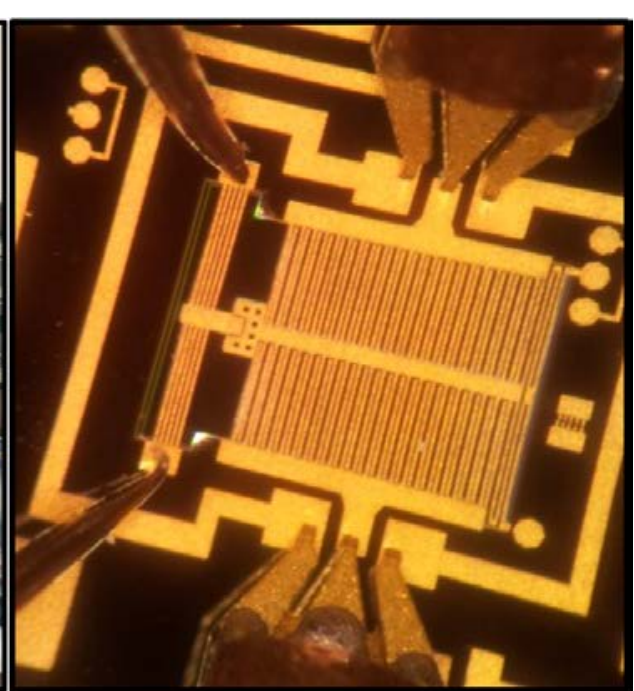

(b)

Figure 5. Experimental setup (a) including HF cables, network analyzer, probe station, dc cables and dc power supply; probe connections (b) including dc probes on the left and GSG ac probes on the right.

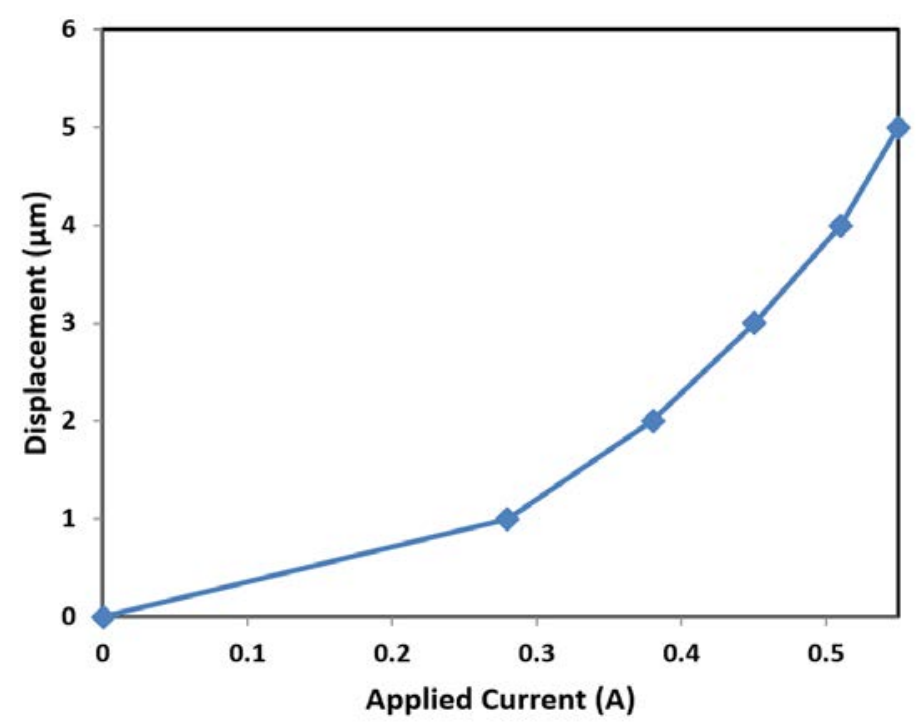

Figure 6. Actuation displacement versus applied current relationship for Chevron-shaped electro-thermal actuator.

To tune the capacitance, the gap between fixed and movable capacitor combs changes by applying dc 
currents through the Chevron-shaped electro-thermal actuator using two dc probes, as illustrated on the left in figure 5 (b). The displacements of the actuation can be observed by on-chip displacement markers from 0 to $5 \mu \mathrm{m}$ with $1 \mu \mathrm{m}$ increments. As shown in figure 6, the actuator moves from 0 to $5 \mu \mathrm{m}$ with the applied current from 0 to $0.55 \mathrm{~A}$. Correspondingly, the capacitor gap changes from initial $6 \mu \mathrm{m}$ to $1 \mu \mathrm{m}$. Therefore, large capacitance change is expected because the capacitance is inversely proportional to the gap between capacitor plates. It is noticed that the actuation voltage is $0.72 \mathrm{~V}$ with $0.55 \mathrm{~A}$ current, therefore a power consumption of 0.396 Watt is required to achieve $5 \mu \mathrm{m}$ displacement. Thus, the device is suitable for low actuation voltage applications where power consumption is of less importance compared to voltage. No plastic deformation in the actuation structures was observed under the actuation current of $0.55 \mathrm{~A}$, indicating that the actuator's temperature is lower than the plastic deformation temperature of nickel material $\left(350^{\circ} \mathrm{C}\right)$.

In order to characterize high frequency parameters such as capacitance, impedance and Q-factor of the tunable capacitor, S-parameters need to be measured through the vector network analyzer connected with ground-signal-ground (GSG) probes, as shown on the right in figure 5 (b). The errors and parasitic in cables and probes were initially characterized and removed by open, short, load and through calibration using Agilent's on-wafer calibration tool of impedance standard substrate (ISS). The parasitic capacitance of the on-chip pads were de-embedded through the procedures given by [20]. After removed all errors and parasitics, the $S_{21}$ parameter of the tunable capacitor was measured and recorded from $10 \mathrm{MHz}$ to $2 \mathrm{GHz}$. Then, the capacitance, Q-factor and impedance of tunable capacitor can be calculated by using equations (2), (3) and (4).

For a fair comparison between the floating design and the conventional (non-floating) design, the same device was characterized with two different probe arrangements: floating configuration (figure 2a) and nonfloating configuration (figure. 2b). In both configurations, the first GSG probe was connected to the fixed plate at point A. The second GSG probe was connected to the fixed plate at point B in the floating configuration, and connected to the anchor pad at point $\mathrm{C}$ in the non-floating configuration.

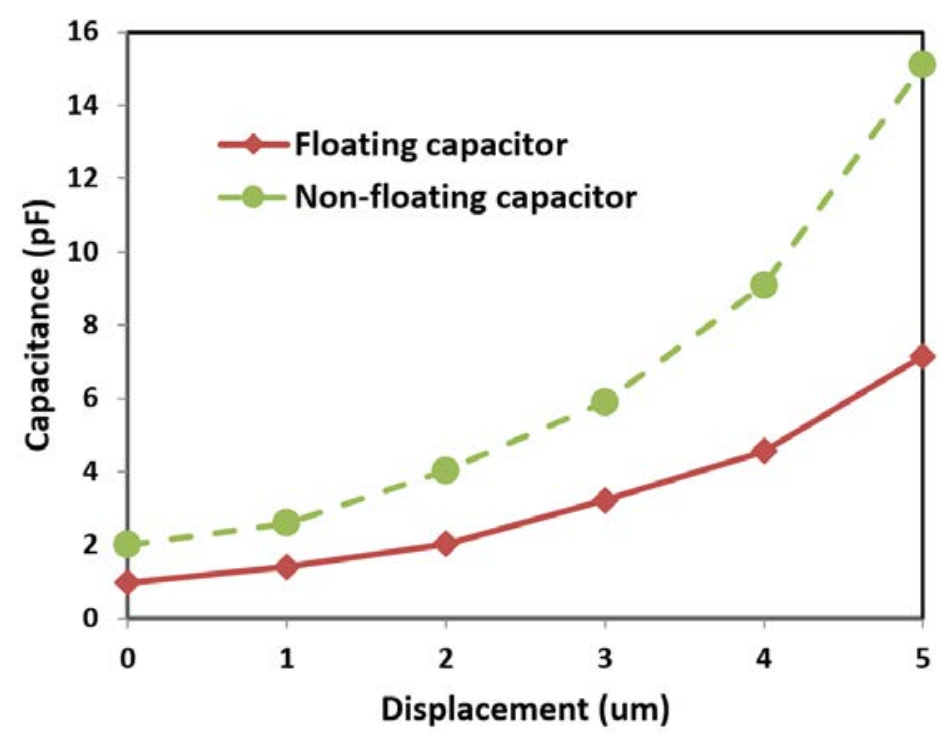

Figure 7. Measured capacitance versus displacement relationships for floating capacitor and traditional non-floating capacitor at $10 \mathrm{MHz}$.

The tunable capacitor is tuned by actuating the Chevron-shaped actuator and changing the gap between fixed and movable comb fingers. The S-parameters at different displacement positions were measured using vector network analyzer. Figure 7 shows the capacitance versus displacement relationship of the floating 
and non-floating tunable capacitors. By applying electrical current through the electro-thermal actuator, the gap between capacitor fingers changes from the initial $6 \mu \mathrm{m}$ to $1 \mu \mathrm{m}$. Consequently, the capacitance measured at $10 \mathrm{MHz}$ increased from $0.98 \mathrm{pF}$ to $7.16 \mathrm{pF}$, which is corresponding to a tuning range of $631 \%$ based on the equation (1). The capacitance and tuning range can be increased further if needed by increasing the actuation current to achieve less than $1 \mu \mathrm{m}$ gap. It is noted that the capacitance of electrically floating tunable capacitor is half of the capacitance of non-floating capacitor as implicated in figure 7 . This can be explained by using the electronic circuit diagram in figure 8. Two identical capacitors $C_{A C}$ and $C_{C B}$ are connected in series in floating capacitor configuration, whereas only one capacitor $C_{A C}$ exists in the nonfloating capacitor configuration. Therefore,

$$
C_{A B}=\frac{1}{2} C_{A C}
$$

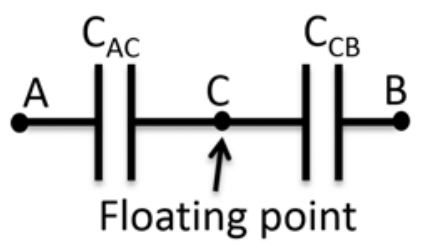

\section{(a)}

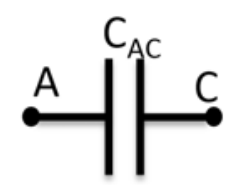

(b)

Figure 8. Schematic circuit diagrams of (a) floating capacitor $C_{A B}$, and (b) non-floating capacitor $C_{A C}$.

The measured Q-factors of floating and non-floating tunable capacitors are shown in figure 9. The Qfactor of floating plate tunable capacitor is initially more than 200 at $100 \mathrm{MHz}$ and decreases to 24.4 at 1 $\mathrm{GHz}$, whereas the Q-factor of non-floating traditional tunable capacitor is 18 at $100 \mathrm{MHz}$ and reduces to 4.4 at $1 \mathrm{GHz}$. The higher measured Q-factor in floating capacitor is due to the spring's resistance loss being eliminated in the RF signal path.

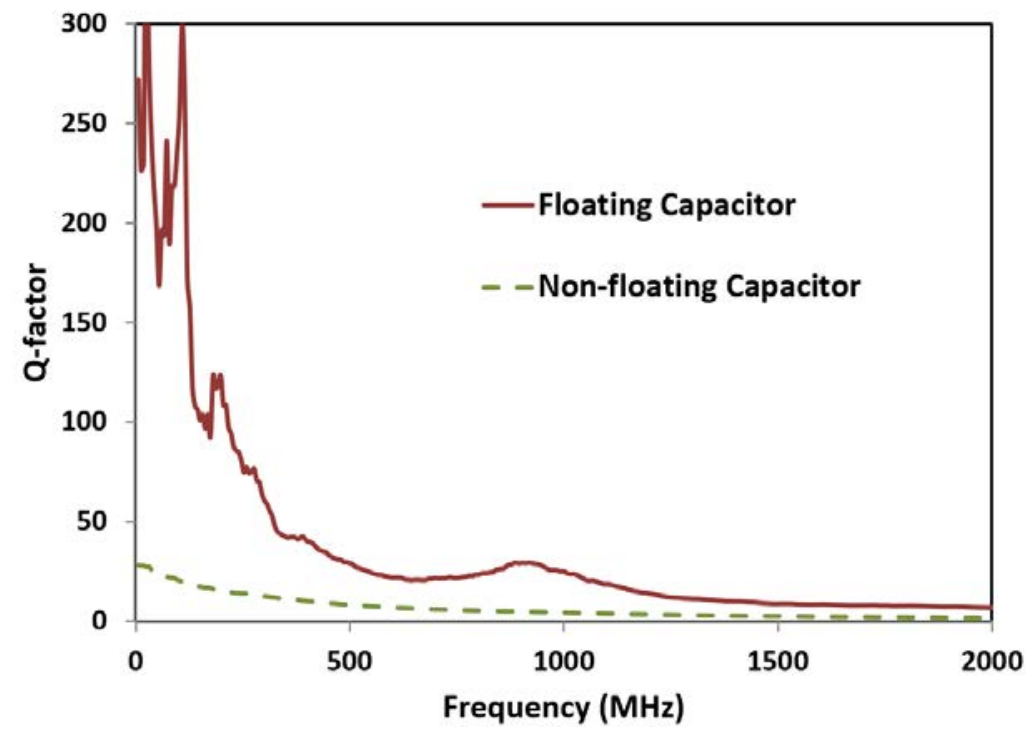

Figure 9. Measured quality factors versus frequency relationships for floating capacitor and traditional nonfloating capacitor. 


\section{Conclusion}

A novel single-layer micromachined tunable capacitor with an electrically floating plate has been proposed and fabricated in a low-cost Metal-MUMPs ${ }^{\mathrm{TM}}$ standard process. We have experimentally demonstrated that the Q-factor of electrically floating tunable capacitor was 5.5 times higher than that of the non-floating tunable capacitor at $1 \mathrm{GHz}$. This improvement was achieved thanks to the elimination of resistance loss in suspension beams of non-floating tunable capacitors. The capacitors were tuned in-plane by electro-thermal actuator without pull-in effect, therefore the capacitance tuning range as high as $631 \%$ has been achieved. The tunable capacitor can be applied in low-loss RF applications, such as VCOs, filters and phase shifters.

\section{Acknowledgement}

This work was partially supported by Griffith University Research Infrastructure Program and was performed in part at the Queensland Node of the Australian National Fabrication Facility (ANFF), a company established under the National Collaborative Research Infrastructure Strategy to provide nano and micro-fabrication facilities for Australia's researchers.

\section{References}

[1] C.-C. Nguyen, "Frequency-selective MEMS for miniaturized communication devices," in Aerospace Conference, 1998 IEEE, 1998, pp. 445-460.

[2] R. L. Borwick III, P. A. Stupar, J. DeNatale, R. Anderson, C. Tsai, K. Garrett, et al., "A high Q, large tuning range MEMS capacitor for RF filter systems," Sensors and Actuators A: Physical, vol. 103, pp. 33-41, 2003.

[3] C. L. Goldsmith, A. Malczewski, Z. J. Yao, S. Chen, J. Ehmke, and D. H. Hinzel, "RF MEMS variable capacitors for tunable filters," International Journal of RF and Microwave Computer-Aided Engineering, vol. 9, pp. 362374, 1999.

[4] W. Robins, Phase noise in signal sources: theory and applications vol. 9: IET, 1984.

[5] Z. Xiao, W. Peng, R. Wolffenbuttel, and K. Farmer, "Micromachined variable capacitors with wide tuning range," Sensors and Actuators A: Physical, vol. 104, pp. 299-305, 2003.

[6] J. Zou, C. Liu, J. Schutt-Aine, J. Chen, and S.-M. Kang, "Development of a wide tuning range MEMS tunable capacitor for wireless communication systems," in Electron Devices Meeting, 2000. IEDM'00. Technical Digest. International, 2000, pp. 403-406.

[7] A. Mehdaoui, M. Pisani, D. Tsamados, F. Casset, P. Ancey, and A. Ionescu, "MEMS tunable capacitors with fragmented electrodes and rotational electro-thermal drive," Microsystem Technologies, vol. 13, pp. 1589-1594, 2007.

[8] J.-B. Yoon and C.-C. Nguyen, "A high-Q tunable micromechanical capacitor with movable dielectric for RF applications," in Electron Devices Meeting, 2000. IEDM'00. Technical Digest. International, 2000, pp. 489-492.

[9] Y. Zhu, M. Yuce, and S. Moheimani, "A low-loss MEMS tunable capacitor with movable dielectric," in Sensors, 2009 IEEE, 2009, pp. 651-654.

[10] H. S. Lee, Y. J. Yoon, D.-H. Choi, and J.-B. Yoon, "High-Q, tunable-gap MEMS variable capacitor actuated with an electrically floating plate," in Micro Electro Mechanical Systems, 2008. MEMS 2008. IEEE 21st International Conference on, 2008, pp. 180-183.

[11] Y. J. Yoon, H. S. Lee, and J.-B. Yoon, "MEMS variable capacitor actuated with an electrically floating plate," in Electron Devices Meeting, 2007. IEDM 2007. IEEE International, 2007, pp. 431-434.

[12] J. R. Reid, V. M. Bright, and J. H. Comtois, "Force measurements of polysilicon thermal microactuators," in Micromachining and Microfabrication'96, 1996, pp. 296-306.

[13] M. J. Sinclair, "A high force low area MEMS thermal actuator," in Thermal and Thermomechanical Phenomena in Electronic Systems, 2000. ITHERM 2000. The Seventh Intersociety Conference on, 2000.

[14] A. Dec and K. Suyama, "Micromachined electro-mechanically tunable capacitors and their applications to RF IC's," Microwave Theory and Techniques, IEEE Transactions on, vol. 46, pp. 2587-2596, 1998. 
[15] V. Jaecklin, C. Linder, N. De Rooij, J. Moret, R. Bischof, and F. Rudolf, "Novel polysilicon comb actuators for xy-stages," in Micro Electro Mechanical Systems, 1992, MEMS'92, Proceedings. An Investigation of Micro Structures, Sensors, Actuators, Machines and Robot. IEEE, 1992, pp. 147-149.

[16] J. Pal, Y. Zhu, J. Lu, D. V. Dao, and F. Khan, "RF MEMS switches for smart antennas," Microsystem Technologies, pp. 1-9, 2014.

[17] R. Dosoudil, "Determination of Permeability from Impedance Measurement using Vector network analyzer.," Journal of Electrical Engineering vol. 63, pp. 97-101, 2012.

[18] A. Cowen, B. Dudley, E. Hill, M. Walters, R. Wood, S. Johnson, et al., "MetalMUMPs design handbook," MEMSCAP Inc., Durham, 2002.

[19] A. Cao, J. Kim and L. Lin, "Bi-directional electrothermal electromagnetic actuators", J. Micromech. Microeng., Vol. 17, pp.975-982, 2007.

[20] M. Koolen, J. Geelen, and M. Versleijen, "An improved de-embedding technique for on-wafer high-frequency characterization," in Bipolar Circuits and Technology Meeting, 1991., Proceedings of the 1991, 1991, pp. 188191. 